\title{
Les facteurs pronostiques du décès dans la cirrhose du foie
}

Le développement récent des possibilités thérapeutiques par greffe de foie rend encore plus essentiel l'établissement du pronostic des cirrhoses hépatiques à partir de critères objectifs et simples. Cet article discute ce que devraient être les conditions d'une étude épidémiologique contrôlée fiable. A partir des données des principales études de ce type publiées dans le monde, les auteurs notent que les paramètres pronostiques les plus significatifs sont l'âge, le sexe, l'encéphalopathie et le temps de prothrombine, et ce, quelle que soit la cause de la cirrhose.

\section{Claire Infante-Rivard Jean-Pierre Villeneuve}

\section{ADRESSE}

C. Infante-Rivard : docteur en médecine, docteur ès sciences, professeur agrégé. École de santé au travail, université McGill, 1130, avenue des Pins-Ouest, Montréal, Québec H3A 1A3, Canada.

J.-P. Villeneuve : docteur en médecine, professeur agrégé. Centre de recherche clinique André-Viallet, hôpital Saint-Luc, université

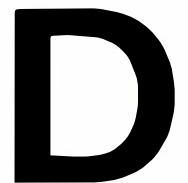

epuis l'avènement de la transplantation hépatique, l'évaluation du pronostic chez les malades atteints de cirrhose est devenue prioritaire. Le choix, souvent difficile, des candidats à une transplantation doit reposer sur une connaissance approfondie de l'évolution de la maladie. Depuis 1980, les études sur le pronostic des cirrhotiques se multiplient donc [1], et plusieurs d'entre elles font appel à des techniques d'analyse multivariée afin de mieux cerner la réalité clinique.

Hormis une publication faite par Schenker, portant sur la cirrhose alcoolique [2], et une autre portant sur le pronostic à court terme (moyenne : sept jours) chez des cirrhotiques hospitalisés pour une hémorragie digestive [3], il n'y a pas eu de revue critique sur l'ensemble des études pronostiques chez les cirrhotiques. C'est ce que nous nous proposons d'étudier dans le présent article, qui présentent un cadre conceptuel permettant de mieux évaluer la qualité des études disponibles.

\section{Objet de l'étude}

Types de cirrhoses. La cirrhose se définit par l'association de trois éléments: une nécrose hépatocytaire (nouvelle ou ancienne), la présence d'une fibrose hépatique diffuse et l'apparition de nodules de régénération. Selon les recommandations de l'Organisation mondiale de la santé (OMS) [4], la cirrhose peut être classée selon des critères morphologiques, histologiques ou étiologiques. La classification étiologique, qui est la plus fréquemment utilisée par les cliniciens, inclut généralement les catégories suivantes de cirrhose: alcoolique, post-hépatitique, cryptogénétique, biliaire primitive, et une catégorie «autre» pour les causes plus rares.

La fréquence des cirrhoses alcooliques dans différents pays est évidemment fonction de l'importance de la consommation d'alcool. En Europe et en Amérique du Nord, on estime que l'alcoolisme est responsable d'environ $50 \%$ des cirrhoses [5]. En Angleterre, cette proportion a été estimée à $52 \%$ [6], alors que dans le département de l'Ille-et-Villaine (France), Péquignot et al. l'estimaient à $90 \%$ [7]. On groupe, sous le terme de cirrhoses post-hépatitiques, les cirrhoses survenant à la suite d'hépatites chroniques virales, autoimmunes, médicamenteuses ou toxiques. La cirrhose biliaire primitive (ou cholangite chronique destructive non suppurée) représente une forme de cirrhose ayant un tableau clinique et évolutif particulier. Un pourcentage important de cirrhoses (25 à $35 \%$ des cas dans la plupart des séries) ne relève apparemment d'aucune étiolo- 
gie précise, et elles sont dites cryptogénétiques ou idiopathiques. Il est probable qu'un bon nombre de ces cas représente le stade évolutif terminal d'une hépatite chronique non $\mathrm{A}$ non B. Enfin, une faible proportion des cirrhoses est due à des maladies hépatiques plus rares (hémochromatose, maladie de Wilson, cirrhose biliaire secondaire, etc.).

Incidence, prévalence et mortalité. Nous ne possédons pas de données significatives sur l'incidence et la prévalence de la cirrhose du foie (Classification internationale des maladies, code 571) dans l'ensemble de la population. Toutefois nous avons des indicateurs indirects de la fréquence de cette maladie. Ces indicateurs proviennent des données de morbidité hospitalière. Ainsi au Canada, entre 1974 et 1979, en moyenne, 69 hommes $/ 100000$ avaient quitté l'hôpital avec un diagnostic de cirrhose ainsi que 33 femmes/100 000 [8].

Aux États-Unis, en 1980, ces chiffres étaient respectivement de 59 et 45 [9]. Il s'agit ici du diagnostic principal, figurant en première position parmi un ensemble de diagnostics possibles. Ces taux sont le reflet de cas incidents (nouveaux), mais aussi de cas anciens (prévalents) qui sont réhospitalisés. Au Canada, la cirrhose ne faisait pas partie des 20 causes les plus fréquentes d'hospitalisation pour l'ensemble de la population.

Au début des années 1980, la cirrhose était la huitième cause la plus importante de décès au Canada [10] et le taux de mortalité, normalisé selon l'âge, était en 1984 de 19,3/100000 chez les hommes et de 8,3/100 000 chez les femmes. En 1985, aux ÉtatsUnis, la cirrhose était la neuvième cause de décès et le taux de mortalité normalisé pour l'âge était de 9,6/100 000 [11].

Survie globale à $\mathbf{5}$ ans. L'évolution des cirrhoses peut varier considérablement d'un individu à l'autre, et l'on doit tenir compte d'éléments étiologiques, cliniques, biologiques et histologiques pour établir un pronostic. Ces éléments seront discutés au paragraphe suivant. Il n'en demeure pas moins que, considérée globalement, la cirrhose est une maladie souvent mortelle. Ainsi, chez $\mathrm{m} / \mathrm{s} n^{\circ} 5$ vol. 5 , mai 89 les sujets atteints de cirrhose alcoolique, dans notre institution, la survie, à cinq ans est d'environ $50 \%$ à partir de l'établissement du diagnostic.

\section{Méthodes d'analyse}

Dans une étude antérieure [1], nous avons proposé une approche méthodologique appropriée aux études pronostiques. Afin d'être valables, les études pronostiques devraient être planifiées, conduites et analysées conformément à des principes méthodologiques empruntés à l'épidémiologie et aux statistiques.

Parmi les principes que nous avons suggérés pour conduire une étude ou évaluer les études publiées, quelquesuns sont si fondamentaux qu'à défaut de les observer, les études ne peuvent prétendre à des conclusions valables.

- Le premier principe porte sur l'utilisation d'une cohorte dite de début d'étude (inception) pour évaluer le rôle des facteurs pronostiques : afin de pouvoir interpréter cliniquement l'incidence de ces facteurs sur la survie des patients, il faut regrouper un nombre donné de patients à un stade spécifique de la maladie (par exemple, au moment du diagnostic ou lors du premier épisode de saignement dû à la présence de varices œesophagiennes). En effet, il ne sera possible d'attribuer, s'il y a lieu, un rôle pronostique à certains paramètres mesurés en fonction des principaux stades de la maladie que si ces facteurs ont été analysés chez des patients au même stade d'évolution. Si les études pronostiques sont entreprises sans tenir compte de l'évolution de la maladie, elles sont vouées à l'échec. Le regroupement de patients au moment de l'hospitalisation est trompeur, certains patients étant recrutés alors qu'ils sont asymptomatiques, d'autres lors d'un épisode de saignement et d'autres, enfin, alors qu'ils sont en insuffisance hépatique terminale. Si certains des facteurs relevés s'avéraient contributifs à la survie dans un groupe aussi hétérogène, il serait impossible pour le clinicien de savoir si ceux-ci jouent un rôle prédictif à chaque stade de la maladie ou à certains stades.

- Un second principe important à respecter dans les études pronosti- ques est la validité des mesures qui sont prises. Les erreurs qui peuvent porter atteinte à la qualité des mesures sont soit de nature aléatoire, soit de nature systématique (ou biais). Des moyens de minimiser ces erreurs consistent en l'utilisation de définitions précises pour les paramètres à mesurer, et de protocoles rigoureux dans la collecte de toutes les informations nécessaires à l'étude pronostique. Ainsi, par exemple, lorsque le but de l'étude est de prédire le décès, il aura été prévu dans le protocole que des cliniciens indépendants établissent la cause du décès, conformément à une procédure déterminée à l'avance et à des définitions explicites des décès suivant les causes. A défaut du respect de ces règles, la confiance que l'on peut avoir dans les résultats d'une étude pronostique est très limitée.

- Un troisième principe concerne la définition de l'échantillonnage de l'étude au sein de la population globale. $A$ priori, on peut mettre en question la représentativité de malades qui viennent tous d'un même centre hospitalier, en particulier d'une unité de soins tertiaires. En effet, les contingents d'une telle unité peuvent varier d'une région à l'autre et encore davantage d'un pays à l'autre. S'il est de plus impossible de déterminer quels sont, parmi les patients d'un même centre hospitalier, ceux qui ont été exclus de l'étude (et pour quel motif), toute possibilité de généralisation des résultats est exclue. Définir l'éligibilité, les critères d'exclusion et les caractéristiques des patients qui n'ont pu être inclus en dépit de leur éligibilité, est un élément essentiel à l'analyse et à la présentation d'une telle étude.

- Une quatrième caractéristique se rapporte à la taille de l'échantillon étudié qui confère aussi un degré plus ou moins grand de certitude aux conclusions de l'étude. Une étude pronostique qui soumettrait à l'analyse statistique plus d'un facteur pronostique pour dix événements dignes d'intérêt (par exemple, dix décès) risquerait de proposer des résultats instables, c'est-à-dire non reproductibles d'une étude à l'autre. - Enfin, puisqu'il est évident que plus d'un paramètre influence le pronostic vital des cirrhotiques, 


\section{RÉFÉRENCES}

1. Infante-RivardC, Villeneuve JP, Esnaola S. Framework for evaluating and conducting prognostic studies: an application to cirrhosis of the liver. J Clin Epidemiol (accepté pour publication).

2. Schenker S. Alcoholic liver disease: evaluation of natural history and prognostic factors. Hepatology $1984 ; 4$ : 365-435.

3. Rueff B. La mesure de la fonction hépatique et le pronostic à court terme des maladies hépatiques. Gastroenterol Clin Biol 1985 ; 9 : 867-70.

4. Anthony PP, Ishak KG, Nayak NC, Poulsen HE, Scheuer PJ, Sobin LH. The morphology of cirrhosis. Recommendations on definition, nomenclature and classification by a working group sponsored by the World Health Organization. J Clin Pathol 1978 ; 31 : 395-414.

5. Lelbach WK Cirrhosis in the alcoholic and its relation to the volume of alcohol abuse. Ann NY Acad Sci 1975 ; 252 : 85-105.

6. Saunders JB, Walters JRF, Davies $P$, Paton A. A 20-year prospective study of cirrhosis. Br Med J 1981 ; 282 : 263-6.

7. Pequignot G, Tuyns AJ, Berta JL. Ascitic cirrhosis in relation to alcohol consumption. Int J Epidemiol 1978 ; 2 : 113-9.

8. Hill G, Bako G, Ferencai L, Moore K. Mortalité et morbidité hospitalières au Canada, 1974-1978. Mal Chron Canad 1987 ; $7: 80-4$.

9. McCarthy E. National Center for Health Statistics. Impatient utilization of short-stay hospitals by diagnosis, United States 1980. Vital and Health Statistics. Series 13, No 74. DHHS Pub No (PHS) 83-1734. Washington :

Public Health Service, Government Printing toute étude se limitant à des formes d'analyse univariée ne peut offrir qu'une mauvaise approximation de la réalité. C'est pourquoi les résultats de telles études ne seront pas pris en considération lorsqu'on étudiera les connaissances actuelles sur les facteurs pronostiques.

Quoique les études pronostiques portant sur la cirrhose abondent, en particulier depuis le début des années 1980, la plupart ne respectent pas les critères méthodologiques fondamentaux énoncés plus haut. Voilà pourquoi les résultats de ces études sont souvent contradictoires. Toutefois, l'état actuel des connaissances reposant sur ces études, nous y puiserons le matériel pour faire le point sur les facteurs pronostiques de la cirrhose. La confiance que nous accordons à ces informations, en fonction de l'observation des critères méthodologiques discutés plus haut, sera énoncée.

Critères pour l'inclusion des études. Parmi les études publiées entre janvier 1980 et septembre 1987, et portant sur le pronostic des cirrhotiques, nous avons retenu celles qui avaient utilisé une approche multivariée appropriée dans l'analyse des résultats, et qui analysaient la durée de survie ou le statut de survie parmi des cirrhotiques [12-25]. A part les données du groupe de Copenhague [21-23, 25], qui sont toutes tirées de deux essais thérapeutiques médicamenteux, nous n'avons retenu que les études observationnelles, c'est-àdire celles où le pronostic est observé en l'absence d'une intervention thérapeutique expérimentale. Les données du groupe de Copenhague ont été retenues à cause de la qualité exceptionnelle de l'analyse des données.

Analyse des études retenues. Appliquant les critères méthodologiques énoncés plus haut, deux études se sont démarquées des autres par leur qualité globale; les résultats de celles-ci seront présentés séparément. Les résultats de l'étude expérimentale de Copenhague sur les cirrhotiques (autres que ceux atteints de cirrhose biliaire primitive), seront aussi présentés séparément. Quelques études n'ont inclus qu'un seul type de cirrhose : ceci nous paraît très logique pour l'analyse du pronostic, de sorte que ces études seront regroupées.

Selon la disponibilité, le risque relatif ainsi que l'intervalle de confiance à $95 \%$ associé à chaque facteur pronostique seront reproduits. Le risque relatif permet d'établir l'importance du facteur sur la survie. Ainsi, par exemple, un sujet ayant un facteur pronostique dont le risque est établi à 1,8 aura 1,8 fois plus de risque de décéder qu'un sujet n'ayant pas ce facteur. L'intervalle de confiance, lorsqu'il ne chevauche pas la valeur nulle du risque relatif (soit 1), indique que le facteur a été jugé statistiquement significatif. Cet intervalle indique surtout l'importance que peut avoir le facteur pronostique. Une variable peut être codifiée pour l'analyse autrement que par sa présence ou son absence (en général ces catégories prennent respectivement les valeurs 1 et 0 ); par exemple, la variable «allongement du temps de prothrombine, en secondes » peut être codifiée en quatre groupes: 0 2,$0 ; 2,1-3,0 ; 3,1-4,5$ et $>4,5$. Afin d'interpréter avec justesse la signification clinique des risques relatifs dans ces cas, il faut se référer aux modalités de codification que nous ne pouvons pas toutes reproduire ici, faute de place.

En plus du calcul du risque relatif, il aurait été souhaitable de pouvoir établir, pour chaque étude, la probabilité de survie attachée à la valeur de chaque catégorie des facteurs pronostiques étudiés, à un moment donné de l'évolution de la maladie (par exemple, la probabilité de survie un an après une mesure à plus de $17 \mu \mathrm{mol} / \mathrm{l}$ de bilirubine).

Deux raisons principales nous empêchent d'utiliser cette approche idéale. La première est reliée au fait que, dans la presque totalité des études publiées, les patients entrent dans l'étude au moment d'une quelconque hospitalisation, et non pas nécessairement à un moment significatif dans l'histoire de leur maladie, tel celui du diagnostic ou de la survenue d'une complication, par exemple. Ainsi, le pronostic à un temps donné (par exemple, un an après l'hospitalisation) devient très difficile à interpréter, car il s'agit d'une durée qui ne signifie pas la même chose pour chacun des sujets étudiés. 


\begin{tabular}{|c|c|c|c|}
\hline \multicolumn{4}{|c|}{$\begin{array}{c}\text { Tableau I } \\
\text { CLASSIFICATION DE CHILD MODIFIÉE PAR PUGH }\end{array}$} \\
\hline \multirow{2}{*}{ Variable } & \multicolumn{3}{|c|}{ Catégorie } \\
\hline & A & B & C \\
\hline $\begin{array}{l}\text { Ascite } \\
\text { Encéphalopathie* } \\
\text { Albumine sérique }(\mathrm{g} / \mathrm{l}) \\
\text { Bilirubine sérique }(\mu \mathrm{mol} / \mathrm{l}) \\
\text { Temps de prothrombine } \\
\text { (secondes au-dessus } \\
\text { du contrôle) }\end{array}$ & $\begin{array}{c}\text { absent } \\
\text { absente } \\
>35 \\
<34 \\
<4,0\end{array}$ & $\begin{array}{c}\text { léger-modéré } \\
\text { stade I-II } \\
28-35 \\
34-51 \\
4,0-6,0\end{array}$ & $\begin{array}{c}\text { tendu } \\
\text { stade III-IV } \\
<28 \\
>51 \\
>6,0\end{array}$ \\
\hline
\end{tabular}

* Selon Trey, Burns et Saunders. N Engl J Med 1966; 274 : 473-81.

Une deuxième raison nous empêche de recourir à cette approche idéale. Il s'agit du manque d'information contenue dans les publications pour permettre de calculer les probabilités spécifiques attachées à un facteur pronostique. Quand on utilise une analyse univariée (un facteur à la fois), il est facile d'estimer et de montrer les probabilités de survie pour chaque facteur pris individuellement. D'autre part, quand on utilise des modèles où plusieurs facteurs sont considérés simultanément dans la régression, la probabilité de survie associée à un facteur isolé, sans tenir compte des autres facteurs, est plus difficile à estimer. Ainsi, dans le contexte de l'utilisation de modèles multivariés, les auteurs doivent fournir au moins deux types d'information pour permettre l'estimation des probabilités de survie : la valeur des coefficients de régression attachés aux facteurs pronostiques significatifs et, dans le cas de l'utilisation du modèle de Cox, la valeur du risque de base sous-jacent (quand tous les facteurs sont favorables). Schlichting et al. [21] soulignent clairement ce dernier point. Or, parmi les 14 études qui ont été recensées, 11 utilisent le modèle de Cox, mais une seule fournit la valeur du risque sous-jacent [23], et seulement six [12, $13,17,21,23,24]$ des 14 études présentent la valeur des coefficients de régression. Dans les deux études [12, 13] qui se démarquent par leur qualité méthodologique globale, le modèle de Cox est utilisé au niveau de l'analyse: la valeur du risque de base n'est pas rapportée, mais des graphiques sont présentés où cette valeur est intégrée. Ces graphiques permettent une estimation approximative (moyennant certaines autres informations) de la probabilité de survie à un temps $t$ selon chaque facteur, ou de la survie médiane. Ces informations seront présentées plus loin, ainsi que la probabilité de survie à court terme (six mois) pour des profils de facteurs pronostiques (d'après Christensen et al. [23]). Enfin, on présentera la probabilité de survie à deux ans, cinq ans et huit ans pour des patients atteints de cirrhose biliaire primitive et présentant un profil donné de facteurs pronostiques (d'après Christensen et al. [25]).

Ajoutons en terminant que, même si toutes les études avaient fourni toute l'information pertinente, un problème de place aurait sûrement limité la possibilité de présenter, pour chaque étude, les probabilités attachées à chaque critère: en effet, une présentation complète exigerait que la définition de chaque catégorie de chaque facteur fût donnée. Puisque les définitions des catégories varient entre les études, un tableau différent serait nécessaire pour chaque étude.

Dans le texte, nous allons souvent faire référence à la classification de Child ou de Pugh, cette dernière étant une variante de la première. Dans la classification de Pugh (Tableau I), cinq paramètres cliniques et biochimiques sont mesurés : la présence d'ascite et d'encéphalopathie, l'albumine, la bilirubine, et le temps de prothrombine (remplaçant l'évaluation de l'état nutritionnel, que l'on retrouve dans la classification de Child). Selon la valeur observée de ces paramètres, un individu est classé dans une des trois catégories (A, B, C) de l'échelle, la première regroupant les individus avec des valeurs favorables, et la dernière, des individus avec des valeurs défavorables (par exemple, ascite sévère, temps de prothrombine très prolongé, etc.).

Dans cet article, le terme «paramètre » sera utilisé librement comme un synonyme de « variable » ou de « facteur », comme cela est maintenant accepté dans la littérature épidémiologique [26]. Strictement parlant, un paramètre est une constante dans une formule statistique ou dans un modèle.

\section{Deux études majeures}

Deux études [12, 13] se distinguent parmi les autres à cause de leur mérite méthodologique et par l'utilisation d'une cohorte assemblée au moment du diagnostic. Dans ces deux études, les cirrhotiques alcooliques représentaient moins de la moitié des cas, c'est-à-dire $42 \%$ dans l'étude de Ginés et al. [13] et $33 \%$ dans l'étude de D'Amico et al. [12]. Dans cette dernière étude, $18 \%$ des patients avaient une cirrhose posthépatitique, tandis que dans l'étude de Ginés et al., cette proportion n'était que de $8,5 \%$.

D'Amico et al. ont étudié la plus grande série de patients, dont 435 étaient des cirrhotiques compensés et 720 des cirrhotiques décompensés. Les cirrhotiques étaient considérés comme compensés s'ils étaient asymptomatiques ou présentaient des symptômes mineurs telles la dyspepsie ou la fatigabilité. En revanche, les cirrhotiques étaient classés comme décompensés s'ils 
avaient des signes ou symptômes majeurs : de l'ascite, une encéphalopathie, un ictère ou un saignement digestif. Le suivi des patients s'est poursuivi pendant une durée maximale de six ans. Parmi les cas compensés dont l'âge moyen était de 52 ans, cinq facteurs diminuaient significativement la survie. Il s'agit, par ordre d'importance, du sexe mâle (risque relatif $=2,16$; intervalle de confiance $=1,30-3,56)$, de la présence de varices œsophagiennes $(1,38 ; 1,10-1,73)$, de celle d'un antigène $\mathrm{HBs}$ positif $(1,32 ; 1,01-1,71)$, d'un âge croissant $(1,26 ; 1,04-1,54$ pour les 41 à 50 ans, 1,$58 ; 1,08-2,37$ pour les 51 à 60 ans, 2,$00 ; 1,12-3,65$ pour les 61 à 70 ans, et 2,52; 1,16-5,62 pour les plus de 70 ans) et d'un allongement du temps de prothrombine $(1,22 ; 1,03-1,43$ pour une valeur de 2,1 à 3,0 secondes, 1,$48 ; 1,06-2,04$ pour 3,1 à 4,5 secondes, et 1,$81 ; 1,09$ 2,92 pour plus de 4,5 secondes).

Parmi les patients se présentant au moment du diagnostic avec une cirrhose décompensée, plusieurs facteurs ont été identifiés comme utiles pour évaluer la probabilité de décès. Ces facteurs étaient, par ordre d'importance: la présence d'un carcinome hépatocellulaire $(3,66 ; 2,65$ $5,04)$, la présence d'encéphalopathie $(1,99 ; 1,55-2,55)$, une présentation marquée par une hémorragie digestive $(1,52 ; 1,16-?)$, un taux élevé de transaminases SGOT plasmatiques (1,33; $1,02-1,73$ pour un niveau de 40 à $80 \mathrm{UI} / 1$, et 1,$76 ; 1,04-2,99$ pour un niveau de plus de $80 \mathrm{UI} / 1)$, la présence de varices œesophagiennes (1,32; 1,16-1,51), une valeur élevée de gammaglobulines $(1,21 ; 1,05-1,34$ pour 15 à $30 \mathrm{~g} / 1$, et 1,$46 ; 1,10-1,79$ pour plus de $30 \mathrm{~g} / \mathrm{l})$, un temps de prothrombine allongé $(1,16 ; 1,07$ 1,27 pour 2,1 à 3,0 secondes, 1,34 ; $1,14-1,61$ pour 3,1 à 4,5 secondes, et 1,$56 ; 1,22 \cdot 2,04$ pour plus de 4,5 secondes), une consommation d'alcool supérieure à $80 \mathrm{~g}$ par jour qui a continué durant le suivi $(1,56$; $1,03-2,3)$ ou une consommation moindre $(1,16 ; 1,01-1,32)$, un antigène $\mathrm{HBs}$ positif $(1,15 ; 1,01-1,31)$, des taux élevés de gammaglutamyl transpeptidase $(1,14 ; 1,03-1,26$ pour un niveau de 18 à 56 UI/1, 1,$29 ; 1,06$ 1,58 pour un niveau de 57 à $100 \mathrm{UI} / 1$, et 1,$48 ; 1,09-2,0$ pour un niveau abaissés de cholinestérase $(1,11 ; 1,01$ 1,21 pour 1,89 à 1,01 UI, 1,$23 ; 1,02-$ 1,46 pour 1,00 à 0,71 UI, 1,$36 ; 1,03$ 1,76 pour 0,70 à $0,50 \mathrm{UI}$, et 1,51 ; $1,04-2,12$ pour moins de $0,50 \mathrm{UI})$.

Sur le Tableau II, on voit les probabilités de survie à cinq ans pour chacun des facteurs pronostiques significatifs, parmi des cirrhotiques compensés, supposant que tous les autres facteurs sont favorables. Le sexe mâle et un âge de plus de 70 ans au moment du diagnostic sont les deux facteurs qui diminuent le plus cette probabilité de survie. Ces probabilités sont bien sûr conformes à l'importance des risques relatifs associés à ces mêmes facteurs pronostiques. Si chacune des probabilités semble assez optimiste, il ne faut pas oublier que la probabilité globale de survie en fonction de plus d'un facteur diminue beaucoup car, dans ces modèles, les risques sont multiplicatifs.

Dans le Tableau III, les probabilités de survie à deux ans sont présentées parmi les cirrhotiques décompensés. La présence d'un carcinome hépatocellulaire est le facteur qui réduit le plus la probabilité de survie, suivie de la présence d'encéphalopathie et de transaminase SGOT à plus de 80 UI.

Dans le Tableau IV, deux profils de patients ayant une cirrhose compensée ainsi que deux profils de patients dont l'affection est décompensée sont contrastés ; ces profils sont accompagnés de la valeur de l'index pronostique (une échelle qui peut être calculée pour chaque patient en utilisant le coefficient de régression estimé pour la variable et la valeur observée de la variable). Les probabilités de survie associées aux valeurs de l'index pronostique sont tirées des figures 4 et 5 de l'article de D'Amico et al. [12].

Ginés et al. ont effectué le suivi jusqu'à 12 ans chez 293 cirrhotiques compensés dont l'âge moyen était de 50,2 ans (erreur-type $=0,6$ an). Parmi les variables qui contribuaient à discriminer les survivants des non-survivants, on retrouvait le sexe mâle, la phosphatase alcaline, les stigmates hépatiques (télangiectasies, angiomes stellaires et/ou érythème palmaire), la bilirubine, les gammaglobulines, l'âge et le temps de prothrombine.

\begin{tabular}{|c|c|}
\hline $\begin{array}{r}\text { PROBABILITÉ (APPROXIN } \\
\text { SELON DIVERS FACTEURS PRON }\end{array}$ & $\begin{array}{l}\text { URVIE A CINQ ANS } \\
\text { CIRRHOTIQUES COMPENSÉS }\end{array}$ \\
\hline Facteur pronostique & $\begin{array}{l}\text { Probabilité de survie à } \mathbf{5} \text { ans } \\
(\%)^{*}\end{array}$ \\
\hline $\begin{array}{l}\text { Sexe mâle } \\
\text { Varices œsophagiennes (présence) } \\
\text { Antigène HBs positif } \\
\text { Age (années) } \\
\quad 41-50 \\
51-60 \\
61-70 \\
>70\end{array}$ & $\begin{array}{l}78 \\
86 \\
86 \\
87 \\
85 \\
80 \\
76\end{array}$ \\
\hline $\begin{array}{l}\text { Temps de prothrombine prolongé (s } \\
\text { au-dessus du témoin) } \\
2,1-3,0 \\
3,1-4,5 \\
>4,5\end{array}$ & $\begin{array}{l}87 \\
84 \\
82\end{array}$ \\
\hline
\end{tabular}

* Probabilité de survie à cinq ans associée à chaque facteur de façon individuelle, en supposant que tous les autres facteurs sont favorables. 


\begin{tabular}{|c|c|}
\hline \multicolumn{2}{|c|}{$\begin{array}{c}\text { Tableau III } \\
\text { PROBABILITÉ (APPROXIMATIVE) DE SURVIE A DEUX ANS } \\
\text { SELON DIVERS FACTEURS PRONOSTIQUES: CIRRHOTIQUES DÉCOMPENSÉS } \\
\text { (D'APRĖS [12]) }\end{array}$} \\
\hline Facteur pronostique & $\begin{array}{l}\text { Probabilité de survie à } 2 \text { ans } \\
(\%)^{*}\end{array}$ \\
\hline Carcinome hépatocellulaire & 18 \\
\hline Encéphalopathie (présence) & 50 \\
\hline Hémorragie digestive (présence) & 65 \\
\hline $\begin{array}{l}\text { Transaminases SGOT (UI/I) } \\
\quad 40-80 \\
>80\end{array}$ & $\begin{array}{l}70 \\
58\end{array}$ \\
\hline Varices œsophagiennes (présence) & 70 \\
\hline $\begin{array}{l}\text { Gammaglobulines }(g / l) \\
15-30 \\
>30\end{array}$ & $\begin{array}{l}80 \\
66\end{array}$ \\
\hline $\begin{array}{l}\text { Temps de prothrombine prolongé (s } \\
\text { au-dessus du témoin) } \\
2,1-3,0 \\
3,1-4,5 \\
>4,5\end{array}$ & $\begin{array}{l}82 \\
71 \\
66\end{array}$ \\
\hline $\begin{array}{l}\text { Alcool ( }>80 \mathrm{~g} / \text { jour) } \\
\text { non (quantité moindre) } \\
\text { oui (ininterrompue) }\end{array}$ & $\begin{array}{l}82 \\
71\end{array}$ \\
\hline Antigène HBs positif & 82 \\
\hline $\begin{array}{l}\gamma \text {-glutamyl transpeptidase }(\mathrm{UI} / \mathrm{I}) \\
28-56 \\
57-100 \\
>100\end{array}$ & $\begin{array}{l}81 \\
75 \\
68\end{array}$ \\
\hline $\begin{array}{l}\text { Cholinestérase (UI/I) } \\
1,89-1,01 \\
1,00-0,70 \\
0,70-0,50 \\
<0,50\end{array}$ & $\begin{array}{l}85 \\
80 \\
72 \\
65\end{array}$ \\
\hline
\end{tabular}

* Probabilité de survie à deux ans associée à chaque facteur de façon individuelle, en supposant que tous les autres facteurs sont favorables.

Dans le Tableau $V$, on présente le risque relatif de décès associé à quelques valeurs des facteurs pronostiques jugés significatifs. Pour estimer chaque risque, il faut se référer aux formules présentées au niveau des pages 124 à 126 de l'article. Le risque d'un individu est estimé en le comparant à la moyenne pour l'ensemble du groupe (donc relativement à la moyenne) ; ainsi, avoir 60 ans - par comparaison à l'âge moyen du groupe (50,2 ans) - est associé à un risque relatif de décès de 1,27 $\mathrm{m} / \mathrm{s} n^{\circ} 5 \mathrm{vol} .5$, mai 89 pas fournies dans l'article.

Parmi les variables considérées dans les deux études, concernant nécessairement les cirrhotiques compensés (car Ginés n'a étudié que ceux-là), certaines se sont avérées contributives dans l'une et pas dans l'autre : ainsi, la bilirubine, les gammaglobulines et la phosphatase alcaline n'étaient pas contributives dans l'étude de D'Amico et al. [12] et la présence de l'antigène $\mathrm{HBs}$ ne comportait pas de valeur pronostique dans l'étude de Ginés et al. [13]. La différence entre ces résultats doit probablement être attribuée au fait que les deux études n'ont pas considéré le même ensemble de variables pronostiques potentielles au départ.

\section{L'étude de Copenhague}

Entre 1962 et 1969, 532 patients ayant une cirrhose prouvée histologiquement, et quelle qu'en soit l'étiologie, ont été inclus dans un essai thérapeutique contrôlé comparant l'efficacité de la prednisone à un placebo, sur la survie. Plusieurs analyses pronostiques utilisant les données de cette étude ont été publiées [21-23]. Malheureusement, l'étude comporte certaines des faiblesses auxquelles nous faisions référence plus haut. Il s'agit d'un groupe de patients non représentatifs de l'ensemble des patients cirrhotiques, ce qui impose une limite à ces analyses, par ailleurs très sophistiquées d'un point de vue quantitatif. En effet, le seul fait d'accepter de participer à une étude et de se plier aux exigences qu'elle requiert pendant un maximum de 12 ans, démarque ces patients de l'ensemble des cirrhotiques sans que l'on puisse dire comment leurs caractéristiques particulières ont interagi avec les facteurs pronostiques à l'étude. De plus, plusieurs paramètres histologiques rarement mesurés de façon routinière ont été soumis à l'analyse, de sorte qu'il est impossible de comparer certains résultats avec ceux d'autres études. Enfin, il s'agissait de patients traités de façon régulière, ce qui n'est pas le cas dans l'ensemble des études pronostiques publiées. Un des atouts de l'étude réside dans la mesure répétée des paramètres cliniques, biochimiques et autres permettant un modèle d'analyse dynamique, certainement 
plus représentatif de la réalité clinique qu'un modèle utilisant une mesure initiale unique.

Dans ce groupe de patients hétérogènes du point de vue de l'histoire naturelle et de l'étiologie, les variables suivantes se sont avérées pronostiques par rapport à la survie jusqu'à 16 ans [23]: un temps de prothrombine allongé, la présence d'ascite, la présence d'un saignement gastrointestinal $(4,09 ; 2,87-5,82)$, un âge plus élevé que 60 ans (une personne de 70 ans a un risque de 1,$68 ; 1,42$ 1,98 par rapport à celle de 60 ans ; autrement dit, le risque augmente de 1,05 unité pour chaque année d'âge après 60 ans, c'est-à-dire : $1,05^{10}=$ $1,68)$, la consommation quotidienne d'alcool $(1,52 ; 1,32-1,75$ pour 10 à $50 \mathrm{~g}$, et 3,$52 ; 2,31-5,47$ pour plus de $50 \mathrm{~g})$, la bilirubine $(2,56 ; 1,79-3,64$ pour un niveau de $70 \mu \mathrm{mol} / \mathrm{l}$ ou plus, l'albumine (par exemple, une valeur de $30 \mathrm{~g} / 1$ comparée à $40 \mathrm{~g} / 1$ est associée à un risque relatif de 1,41 ; 1,21-1,65), la phosphatase alcaline (par exemple, une valeur de 30 unités KA par rapport à 10 , est associée à un risque relatif de 1,$48 ; 1,17-1,88$ ), un statut nutritionnel défavorable $(1,75 ; 1,27-2,39)$, et un degré marqué d'inflammation du foie à l'histologie, qui - paradoxalement - était associé à un risque inférieur à 1 $(0,57 ; 0,43-0,57)$. Seuls le temps de prothrombine (calculé par: $\log _{e}[\%$ de normal] - 4) et la présence d'ascite modifiaient l'effet du traitement, c'est-à-dire n'avaient pas le même effet selon que le patient recevait la prednisone ou le placebo (par exemple, un temps de prothrombine de $60 \%$, au lieu de $90 \%$, était associé à un risque relatif de 1,$89 ; 1,59-2,25$ parmi les patients recevant la prednisone et à un risque de 1,$40 ; 1,20$ 1,62 chez ceux qui recevaient le placebo; la présence d'ascite était accompagnée d'un risque relatif de 5,$25 ; 3,22-8,58$ dans le groupe prednisone, et d'un risque de 3,$22 ; 2,05$ 5,05 dans le groupe placebo). On remarquera que les éléments constituant les classifications de Child ou de Pugh, à l'étonnante exception de l'encéphalopathie, font partie des facteurs pronostiques utiles.

Sur le Tableau VI, deux patients sont présentés avec leurs profils pronostiques respectifs. On notera que la la mesure des facteurs pronostiques est très différente entre ces deux patients (on pourrait aussi considérer qu'il s'agit du même patient dont les paramètres cliniques et biochimiques sont mesurés de nouveau à une date ultérieure). Les facteurs qui font que le patient «b» a une faible probabilité de survie à six mois, par comparaison avec le patient «a», sont le développement d'une ascite, une diminution de l'albumine, une augmentation de la bilirubine sérique et de la phosphatase alcaline.
Chacun de ces facteurs est associé, comme on l'a vu précédemment, à un risque relatif plutôt important.

\section{Autres études sur des populations hétérogènes de cirrhotiques}

D'autres études [14-17] sont caractérisées par l'utilisation de populations hétérogènes et par le fait que chacune d'entre elles s'est attardée à étudier la contribution pronostique d'un facteur en particulier. Cepen-

\begin{tabular}{|c|c|c|}
\hline \multicolumn{3}{|c|}{$\begin{array}{l}\text { Tableau IV } \\
\text { RVIE A CINQ ANS ET A DEUX ANS } \\
\text { E PROFILS PRONOSTIQUES : } \\
\text { OMPENSÉS ET DÉCOMPENSÉS } \\
\text { D'APRĖ [12]) }\end{array}$} \\
\hline \multicolumn{3}{|c|}{ CIRRHOTIQUES COMPENSÉS } \\
\hline Facteur & Patient A & Patient B \\
\hline $\begin{array}{l}\text { Sexe } \\
\text { Varices œsophagiennes } \\
\text { Antigène HBs } \\
\text { Age (années) } \\
\text { Prolongation } \\
\text { prothrombine (sec) }\end{array}$ & $\begin{array}{l}\text { homme } \\
\text { présence } \\
\text { absence } \\
50 \\
4,5\end{array}$ & $\begin{array}{c}\text { femme } \\
\text { absence } \\
\text { absence } \\
50 \\
2,5\end{array}$ \\
\hline Index pronostique & 4,4 & 1,5 \\
\hline $\begin{array}{l}\text { Probabilité de survie à } 5 \text { ans } \\
\text { (\%) (approximative) }\end{array}$ & 60 & 85 \\
\hline
\end{tabular}

\begin{tabular}{|c|c|c|}
\hline \multicolumn{3}{|c|}{ CIRRHOTIQUES DEECOMPENSÉS } \\
\hline Facteur & Patient A & Patient B \\
\hline $\begin{array}{l}\text { Hépatome } \\
\text { Encéphalopathie } \\
\text { Hémorragie } \\
\text { Transaminases SGOT (UI/I) } \\
\text { Varices œsophagiennes } \\
\text { Gammaglobulines (g/l) } \\
\text { Prolongation prothrombine } \\
\text { (sec) } \\
\text { Alcool (> } 80 \mathrm{~g} / \text { jour) } \\
\gamma \text {-glutamyl transférase (UI/I) } \\
\text { Cholinesterase (UI/I) }\end{array}$ & $\begin{array}{c}\text { absent } \\
\text { présence } \\
\text { absence } \\
70 \\
\text { absence } \\
15 \\
2,5 \\
\text { oui } \\
30 \\
1,5\end{array}$ & $\begin{array}{c}\text { absent } \\
\text { présence } \\
\text { absence } \\
45 \\
\text { absence } \\
13 \\
2 \\
\text { non } \\
30 \\
1,9\end{array}$ \\
\hline Index pronostique & 7,7 & 3,3 \\
\hline $\begin{array}{l}\text { Probabilité de survie à } 2 \text { ans } \\
\text { (\%) (approximative) }\end{array}$ & 0 & 20 \\
\hline
\end{tabular}




\begin{tabular}{|c|c|c|c|}
\hline \multicolumn{4}{|c|}{$\begin{array}{c}\text { Tableau V } \\
\text { SURVIE MÉDIANE (APPROXIMATIVE) EN JOURS, } \\
\text { SELON DIVERS FACTEURS PRONOSTIOUES : CIRRHOTIQUES COMPENSÉS } \\
\text { (D'APRES [13]) }\end{array}$} \\
\hline \multicolumn{2}{|l|}{ Facteur pronostique } & $\begin{array}{l}\text { Index pronostique } \\
\text { (ou risque relatif) }\end{array}$ & $\begin{array}{c}\text { Survie médiane } \\
\text { en jours }\end{array}$ \\
\hline $\begin{array}{l}\text { Bilirubine } \\
\text { ( } \mu \mathrm{mol} / \mathrm{I})\end{array}$ & $\begin{array}{r}5 \\
10 \\
20\end{array}$ & $\begin{array}{l}0,71 \\
0,98 \\
1,84\end{array}$ & $\begin{array}{l}2100 \\
1900 \\
1400\end{array}$ \\
\hline Gammaglobulines (g/l) & $\begin{array}{l}15 \\
18 \\
25\end{array}$ & $\begin{array}{l}0,83 \\
1,04 \\
1,33\end{array}$ & $\begin{array}{l}2200 \\
1900 \\
1700\end{array}$ \\
\hline Stigmates hépatiques & $\begin{array}{l}\text { absence } \\
\text { présence }\end{array}$ & $\begin{array}{l}0,86 \\
1,22\end{array}$ & $\begin{array}{l}2200 \\
1600\end{array}$ \\
\hline Temps de prothrombine (\%) & $\begin{array}{l}95 \\
85 \\
70\end{array}$ & $\begin{array}{l}0,83 \\
0,99 \\
1,33\end{array}$ & $\begin{array}{l}2200 \\
1900 \\
1700\end{array}$ \\
\hline Sexe & $\begin{array}{l}\text { féminin } \\
\text { masculin }\end{array}$ & $\begin{array}{l}0,62 \\
1,24\end{array}$ & $\begin{array}{l}2600 \\
1600\end{array}$ \\
\hline Age (années) & $\begin{array}{l}40 \\
50 \\
60\end{array}$ & $\begin{array}{l}0,78 \\
1,00 \\
1,27\end{array}$ & $\begin{array}{l}2200 \\
1900 \\
1700\end{array}$ \\
\hline Phosphatase alcaline & $\begin{array}{l}\text { normale } \\
\text { élevée }\end{array}$ & $\begin{array}{l}0,79 \\
1,21\end{array}$ & $\begin{array}{l}2200 \\
1600\end{array}$ \\
\hline
\end{tabular}

Mannes et al. [15] ont étudié le rôle des acides biliaires sériques dans un groupe de 77 patients dont 55 (71 \%) avaient une cirrhose alcoolique et les autres, une cirrhose post-hépatitique. Le statut de survie a été évalué à un an. Seuls les acides biliaires sériques et les signes d'encéphalopathie étaient déterminants par rapport au statut de décès ou de survie, un an après le début de l'étude. Les éléments de la classification de Child (albumine, bilirubine, encéphalopathie, ascite et état nutritionnel) ainsi que la pseudocholinestérase, le temps de prothrombine et l'âge étaient candidats dans l'analyse pronostique multivariée. On notera que la $\gamma$-glutamyl transpeptidase, accessoirement utilisée dans l'étude de Poynard et al. [14], a été jugée sans utilité pronostique dans l'étude de Mannes lors des analyses univariées préliminaires.

Villeneuve et al. [16] ainsi qu'Infante-Rivard et al.[17] ont évalué l'utilité de la classification de Pugh (albumine, bilirubine, encéphalopathie, ascite et temps de prothrombine) comme outil pronostique.

dant, les facteurs pronostiques n'étaient pas tous mesurés au moment du diagnostic (les malades avaient divers type de cirrhose) et elles regroupaient des malades compensés comme décompensés.

Poynard et al. [14] ont étudié 129 malades, dont 115 - soit $89 \%$ avaient une cirrhose alcoolique. Les patients ont été suivis pendant un an, et la survie estimée à ce momentlà. Les résultats de cette étude suggéraient que, compte tenu de la présence d'encéphalopathie et d'ascite, la bilirubine sérique et, de façon marginale, la $\gamma$-glutamyl transpeptidase $(\gamma \mathrm{GT})$ se révélaient utiles au pronostic de survie. Les autres facteurs qui avaient été pris en considération mais non retenus comme contributifs étaient le temps de prothrombine, l'albumine, les transaminases SGOT, le rapport bilirubine sérique $/ \gamma$-glutamyl transpeptidase, l'âge et le saignement gastro-intestinal.

$\mathrm{m} / \mathrm{s} n^{\circ} 5$ vol. 5 , mai 89

\begin{tabular}{|c|c|c|}
\hline \multicolumn{3}{|c|}{$\begin{array}{c}\text { PROBABILITÉ DE SURVIE A SIX MOIS EN FONCTION DE PROFILS } \\
\text { DE FACTEURS PRONOSTIQUES : } \\
\text { CIRRHOTIQUES COMPENSÉS ET DÉCOMPENSÉS } \\
\text { (D'APRES [23]) }\end{array}$} \\
\hline Facteur pronostique & Patient A & Patient B \\
\hline $\begin{array}{l}\text { Index de prothrombine (\% de normal) } \\
\text { Ascite } \\
\text { Saignement gastro-intestinal } \\
\text { Age au début de l'étude (années) } \\
\text { Consommation d'alcool (g/jour) } \\
\text { Bilirubine ( } \mu \mathrm{mol} / \mathrm{l}) \\
\text { Albumine (g/I) } \\
\text { Infiltrat inflammatoire à l'histologie hépatique } \\
\text { Mauvais état nutritionnel } \\
\text { Phosphatase alcaline (unités KA/I) }\end{array}$ & $\begin{array}{l}59 \\
\text { absent } \\
\text { non } \\
61 \\
>50 \\
17 \\
42,6 \\
\text { oui } \\
\text { non } \\
7,8\end{array}$ & $\begin{array}{l}50 \\
\text { modéré } \\
\text { non } \\
61 \\
10-50 \\
120 \\
26,6 \\
\text { oui } \\
\text { non } \\
39,8\end{array}$ \\
\hline \multicolumn{3}{|l|}{ Probabilité de survie à 6 mois (\%) } \\
\hline $\begin{array}{l}\text { Groupe prednisone } \\
\text { Groupe placebo }\end{array}$ & $\begin{array}{l}92 \\
92\end{array}$ & $\begin{array}{l}12 \\
34\end{array}$ \\
\hline
\end{tabular}




\section{RÉFÉRENCES}

10. Ku P, Smith E, MaoY. Tendances récentes de la mortalité au Canada. Mal Chron Canad 1986; 7 : 50-3.

11. National Center for Health Statistics. Advance report of final mortality Statistics 1985. Monthly Vital Statistics Report vol. 6, No 5, Suppl DHHS Pub No (PHS) 87-1120. Hyattsville : Public Health Service, MD, 1987.

12. D’Amico G, Morabito A, Pagliaro L, Marubini E. The Liver Study Group of «V. Cervello» Hospital. Survival and prognostic indicator in compensated and decompensated cirrhosis. Dig Dis Sci 1986; 31 : 468-75.

13. Ginés P, Quintero E, Arroyo V, et al. Compensated cirrhosis : natural history and prognostic factors. Hepatology 1987; 7 : 122-8.

14. Poynard T, Zourabichvili O, Hilpert G, et al. Prognostic value of total serum bilirubin/ gamma-glutamyl transpeptidase ratio in cirrhotic patients. Hepatology 1984 ; 4 : 324-7.

15. Mannes GA, Thieme C, Stellaard F, Wang T, Sauerbruch T, Paumgartner G. Prognostic significance of serum bile acid in cirrhosis. Hepatology 1986 ; 5 : 928-31.

16. Villeneuve JP, Infante-Rivard C, A mpelas M, Pomier-Layrargues G, Huet PM, Marleau D. Prognostic value of the aminopyrine breath-test in cirrhotic patients. Hepatology $1986 ; 5: 928-31$.

17. Infante-Rivard C, Esnaola S, Villeneuve JP. Clinical and statistical validity of conventional prognostic factors in predicting short-term survival among cirrhotics. Нераtology $1987 ; 8: 660-4$.

18. Orrego H, Israel Y, Blake JE, Medline A. Assessment of prognostic factors in alcoholic liver disease: toward a global quantitative expression of severity. Hepatology 1983 ; 3 :
Dans la première étude [16], dont la durée du suivi dépassait largement un an, on démontrait qu'un test sophistiqué, le taux d'exhalaison de l'aminopyrine, n'apportait rien de plus à la valeur pronostique de la classification de Pugh, qui peut être obtenue dans un contexte clinique usuel. Dans la seconde étude [17], on mettait en évidence le fait qu'à eux seuls, les éléments de la classification de Pugh pouvaient prédire correctement le décès à court terme chez $80 \%$ des patients. L'encéphalopathie sévère $(14,98 ; 10,52-21,32)$ et l'ascite sévère $(8,06 ; 3,63-61,68)$ avaient des contributions individuelles significatives, tandis que celles de l'albumine et du temps de prothrombine ne l'étaient que marginalement. Le risque de décès augmentait à 2,$48 ; 0,99$ 6,24 pour chaque diminution de $10 \mathrm{~g} / \mathrm{l}$ du taux d'albumine, et il augmentait à 1,$36 ; 0,99-1,85$ pour chaque seconde du temps de prothrombine au-dessus du témoin.

\section{Études portant sur des malades avec cirrhose alcoolique}

Trois études multivariées récentes n'incluant que des cirrhotiques alcooliques retiennent l'attention. Chacune d'entre elles présente malheureusement plusieurs des défauts décrits précédemment, de sorte que la confiance qu'on peut avoir dans les résultats qu'elles proposent est limitée. Néanmoins, ce sont les études les plus informatives à l'heure actuelle. Orrego et al. [18] ont analysé la contribution de paramètres cliniques, d'épreuves de laboratoire et de données histologiques à la prédiction du décès parmi 253 alcooliques, un an après le début de l'étude. Les niveaux d'hémoglobine (en pourcentage de la normale), d'albumine et la présence d'encéphalopathie étaient des prédicteurs indépendants de la mortalité. Naveau et al. [19] évaluaient, parmi 160 patients alcooliques, le rôle de la fibronectine sérique en compagnie de quelques autres paramètres cliniques (âge, encéphalopathie et ascite) et biochimiques (albumine, bilirubine sérique, gammaglutamyl transférase, le rapport bilirubine sérique $/ \gamma$-glutamyl transférase et le temps de prothrombine) sur la survie à un an.
Comme dans l'étude d'Orrego et al. [18], l'encéphalopathie et l'albumine s'avéraient des variables discriminantes, mais aussi la fibronectine sérique et l'âge. Il est à noter que dans leur étude antérieure [14], où $89 \%$ des patients étaient des alcooliques, la bilirubine et la $\gamma$-glutamyl transférase étaient des variables importantes. Enfin, le même groupe d'auteurs étudiait la valeur pronostique à deux ans de survie de 23 variables parmi 249 cirrhotiques alcooliques [20]. Les variables contributives étaient : l'encéphalopathie ( 1,56 pour désorientation et 2,44 pour coma, chacune par rapport à l'absence d'encéphalopathie), l'albumine (une augmentation du risque de 1,97 pour chaque diminution de $10 \mathrm{~g} / \mathrm{l}$ du taux d'albumine), l'âge (une augmentation du risque de 1,05 pour chaque année d'âge en plus), la bilirubine totale (une élévation du risque de 2,81 par $\mu \mathrm{mol} / \mathrm{l}$ d'augmentation), et la $\gamma$-glutamyl transférase (le risque augmente de 1,70 pour chaque unité de glutamyltranspeptidase qui diminue).

\section{Les facteurs pronostiques de la cirrhose biliaire primitive (CBP)}

Roll et al. rapportaient en 1983 [24] des données sur le pronostic de 280 patients diagnostiqués entre 1955 et 1979 comme ayant une CBP. De ce groupe, 243 patients (87\%) étaient symptomatiques et 37 (13\%) étaient asymptomatiques, l'âge moyen au diagnostic était de 48 ans et $92 \%$ étaient des femmes. L'intérêt de cette étude réside dans le fait que les données cliniques et biologiques qui ont servi à l'étude du pronostic de la survie (jusqu'à 19 ans plus tard) ont été recueillies au moment du diagnostic. Toutefois, pour $70 \%$ des patients, les données rapportées provenaient de médecins consultants, ce qui introduit assurément une variabilité dans les observations. De plus, on peut critiquer le modèle pronostique un peu simpliste, postulant qu'une seule mesure, faite jusqu'à 19 ans auparavant puisse représenter adéquatement la réalité. Les mesures suivantes obtenues au moment du diagnostic se sont avérées utiles pour prédire la survie: l'âge (1,04 par année d'âge), la présence d'hépatomégalie $(2,1)$, un niveau de biliru- 
Tableau VII

PROBABILITÉ DE SURVIE A DEUX ANS, CINO ANS ET HUIT ANS EN FONCTION DE PROFILS DE FACTEURS PRONOSTIQUES : CIRRHOSE BILIAIRE PRIMITIVE (D’APRÈS [25])

\begin{tabular}{|l|c|c|}
\hline \multicolumn{1}{|c|}{ Facteur pronostique } & Patient A & Patient B \\
\hline Bilirubine sérique ( $\mu$ mol/I) & 32 & 70 \\
Age (années) & 68 & 51 \\
Cirrhose & non & oui \\
Albumine (g/I) & 32 & 26 \\
Cholestase centrolobulaire & non & non \\
Traitement par azathioprine & non \\
\hline Probabilité de survie (\%) & & \\
\hline ans & 91 & 71 \\
5 ans & 65 & 21 \\
8 ans & 28 & 2 \\
\hline
\end{tabular}

bine supérieure à $85 \mu \mathrm{mol} / \mathrm{l}(2,26)$ et les signes histologiques de fibrose portale qui seraient protecteurs $(0,26)$. Les variables qui n'ont pas été retenues sont les symptômes cliniques (tels le prurit, la fatigabilité, l'ictère, etc.), l'ascite, la splénomégalie, l'albumine, les immunoglobulines et les autres paramètres de la biopsie hépatique, dont la présence de cirrhose et de cholestase.

Christensen et al. [25] ont étudié l'effet de l'azathioprine sur la survie de 248 patients atteints de CBP. Ils ont aussi analysé la contribution de plusieurs autres facteurs sur la survie. Les patients semblent avoir été inclus dans l'essai thérapeutique au moment du diagnostic. Les limites énoncées plus haut à propos des analyses du groupe de Copenhague s'appliquent ici car il s'agit d'une étude similaire. Les marqueurs importants du pronostic étaient: la bilirubine sérique (une augmentation de la bilirubine de $30,40 \mu \mathrm{mol} / 1$ contre une valeur de 30, est accompagnée d'une augmentation du risque relatif de 2,05, 1,71-2,46), l'âge (une personne de 60 ans a un risque de 1,$26 ; 1,13$ 1,41 par rapport à une autre de 50 ans), la présence de cirrhose $(2,41$; $1,56-3,71)$ et de cholestase centrolobulaire à la biopsie $(1,97 ; 1,16$ $\mathrm{m} / \mathrm{s} n^{\circ} 5$ vol. 5 , mai 89 jamais, ou à peu près, justifier le choix des variables étudiées en se fondant sur une hypothèse explicite. La plupart des études sont teintées d'un opportunisme ponctuel, à savoir la disponibilité d'une base de données et des moyens informatiques pour l'analyser. A la lecture de ces différents travaux, il se dégage une impression de superposition plutôt que de complémentarité. Finalement, quoique plusieurs des mêmes variables aient été analysées d'une étude à l'autre, les résultats ne sont pas cohérents : si certaines de ces variables s'avèrent utiles au pronostic dans la majorité des études, plusieurs autres s'avèrent à la fois utiles dans certaines études et inutiles dans d'autres.

D'autre part, le consensus essentiellement tacite sur la définition des paramètres, même les plus couramment utilisés, devrait être remplacé par un consensus explicite. On pourrait alors comprendre jusqu'à quel point les différentes façons de concevoir les paramètres cliniques particuliers ont eu une influence sur les conclusions des études. Enfin, et c'est probablement le problème le plus aigu, le fait que dans aucune des études analysées il n'y ait eu d'étalonnage des observateurs contribue à la variabilité des observations et à la difficulté de les faire ressortir comme contributives au niveau de l'analyse. Quand on ajoute à ce problème la variabilité inter-centres, on conçoit facilement que les résultats ne soient pas intégralement reproductibles d'une étude à l'autre.

Malgré toutes ces remarques, et parce qu'il faudra continuer à jauger quotidiennement le pronostic des cirrhotiques, un effort doit être fait pour dégager les éléments qui sont cliniquement utiles et qui devraient être inclus dans les études pronostiques futures.

Les variables qui semblent avoir la plus grande valeur pronostique, quelle que soit l'étiologie de la cirrhose, sont l'âge, le sexe, l'encéphalopathie et le temps de prothrombine. D'autres ont été relevées moins fréquemment, mais peuvent difficilement être ignorées. Ce sont : l'ascite, la bilirubine, l'albumine et la présence de varices œsophagiennes.

Pour les cirrhotiques alcooliques, les variables énumérées ci-dessus s'appliqueraient probablement puisque, 


\section{RÉFÉRENCES}

19. Naveau S, Poynard T, Abella A, et al. Prognostic value of serum fibronectic concentration in alcoholic cirrhotic patients. Hepatology $1985 ; 5: 819-23$.

20. Pignon JP, Poynard T, Naveau S, Marteau P, Zourabichvili O, Chaput JC. Analyse multivariée selon le modèle de Cox de la survie des patients atteints de cirrhose alcoolique. Gastroenterol Clin Biol 1986; 10: 461-67.

21. Schlichting $\mathbf{P}$, Christensen E, Andersen PK, et al. and the Copenhagen Study Group for Liver Diseases. Prognostic factors in cirrhosis identified by Cox's regression model. Hepatology 1983 ; 3 : 889-95.

22. Christensen E, Schlichting P, Fauerholdt L, et al. and the Copenhagen Study Group for Liver Diseases. Prognostic value of Child-Turcotte criteria in medically treated cirrhosis. Hepatology 1984 ; 4 : 430-5.

23. Christensen E, Schlichting P, Andersen PK, et al. and the Copenhagen Study Group for Liver Diseases. Updating prognosis and therapeutic effect evaluation in cirrhosis with Cox's multiple regression model for time-dependent variables. Scand J Gastroenterol 1986 ; 21 : 163-74.

24. Roll J, Boyer JL, Barry D, Klatskin G The prognostic importance of clinical and histologic features in asymptomatic and symptomatic primary biliary cirrhosis. $N$ Engl J Med 1983; 308: 1-7.

25. Christensen E, Neuberger J, Crowe J, et al. Beneficial effect of azathioprine and prediction of prognosis in primary biliary cirrhosis. Gastroenterology 1985 ; 89 : 1084-91.

26. Last JM. A dictionary of epidemiology. malgré l'hétérogénéité des populations étudiées, le groupe des alcooliques domine. On aura remarqué que la consommation d'alcool a rarement été considérée au niveau de l'analyse. Cela résulte peut-être de la difficulté à mesurer ce paramètre avec fiabilité. Toutefois, ce paramètre ne peut être ignoré si on se fonde sur les connaissances déjà acquises. Chez les malades atteints de cirrhose biliaire primitive, la bilirubine sérique s'est avérée un marqueur significatif.

Il semble donc que des facteurs simples et dont la mesure est accessible à tous les centres hospitaliers s'avèrent les plus utiles. De plus, une étude [17] a suggéré que ces paramètres pouvaient prédire correctement le sort de $80 \%$ des patients malgré les populations hétérogènes. Cette constatation est encourageante. A un moment où les décisions cliniques sont de plus en plus influencées par le rapport coût-avantage des interventions, il faudra continuer de chercher des indicateurs simples, peu coûteux, acceptables pour les patients, qui nous permettent de rehausser notre capacité de repérer les patients dont le pronostic est soit favorable, soit défavorable à un moment donné après le diagnostic. A la lumière de l'information présentée dans cet article, il est clair que des études de meilleure qualité seront nécessaires pour mieux définir le pronostic des patients cirrhotiques. Les recommandations suivantes s'imposent pour la conduite de ces études: il serait utile d'étudier des groupes diagnostiques homogènes, le choix de variables soumises à l'analyse devrait être justifié, et on ne saurait trop insister pour que les études soient conduites conformément à des principes méthodologiques rigoureux. Il importe de rappeler que le recours aux analyses statistiques complexes ne peut en aucun cas amender une étude dont l'architecture est fautive.

En conclusion, s'il faut pour le futur souhaiter de meilleures études, il faut reconnaître l'apport collectif des études publiées. Il sera intéressant de voir jusqu'à quel point les connaissances qu'elles ont établies seront modifiées par des études plus parfaites du point de vue méthodologique

\section{Summary}

Pronostic factors in cirrhosis of the liver

With the advent of hepatic transplantation, understanding the prognosis of cirrhotic patients has become imperative. In this article, after recalling the etiological types of cirrhosis as well as the morbidity and the mortality figures associated with the disease, a summary framework for conducting and evaluating prognostic studies is proposed. Thereafter, major studies of the last decade are reviewed, underscoring the variables which were contributive to our understanding of prognosis.

\section{TIRÉS A PART}

C. Infante-Rivard. 